\title{
P53 pseudogene: potential role in heat shock induced apoptosis in a rat histiocytoma
}

\author{
Amere Subbarao Sreedhar \\ Centre for Cellular and Molecular Biology, Hyderabad, India; assr@ccmb.res.in
}

Received 1 June 2010; revised 2 July 2010; accepted 5 July 2010.

\begin{abstract}
The p53 tumor suppressor gene is either nonfunctional or highly and frequently mutated in majority of cancers. In our study towards understanding cellular adaptations to stress using a rat histiocytic tumor model, we have identified mis-sense mutation in p53 that led to premature termination of translation at the carboxyl-terminus. Further, the cDNA isolated from heat stressed cells producing two amplicons with cDNA specific primers ( $\mathrm{N}$-terminus) suggested occurrence of possible pseudogene(s). A comparative analysis between different tumor cell lines of rat origin and rat genomic DNA using p53 gene specific primers resulted in the amplification of a processed pseudogene and its positive interaction with wild type p53 probe on Southern blot analysis. The genomic DNA sequence analysis, and sequence comparison with cDNA discovered that the processed pseudogene lacks DNA binding domain and nuclear localization signal, however, contains the ribosomal entry and stop signals. Rat genome BLAST analysis of the pesudogene suggested chromosome-18 localization which was in addition to $14,13,10,9$ localization of the cDNA. In the interest of unraveling hidden dimensions of p53 tumor suppressor gene, our study explores the probability of p53 functional pseudogenes in rat histiocytoma.
\end{abstract}

Keywords: Pseudogene; P53; Tumor; Rat Histiocytoma

\section{INTRODUCTION}

The tumor suppressor p53 is a multifunctional protein that is involved in a variety of biological processes such as growth arrest, apoptosis, differentiation and senescence $[1,2]$. Aberrant expression of this gene results in either a gain of transforming potential or a loss in tumor suppressor activity $[3,4]$. The p53 gene mutation, deletion, insertion or protein sequestration etc are often found in many cancers $[5,6]$ and these mutations affect the p53 binding to DNA [7]. Analysis of the degeneracy of p53 DNA-binding site suggests that there may be as many as $200-400$ p53 target sequences or perhaps more [8]. Despite the high frequency with which p53 is mutated during tumor development, a substantial proportion of tumors still express the wild type p53 [9]. This could be the reason in spite of exhaustive information on p53 modifications the corresponding role of p53 modification in experimental animal tumor models is poorly understood.

We are investigating the role of p53 in heat stress-induced rat histiocytic tumors models. In the process of elucidating heat stress induced cell death pathways and evaluating the functional significance of p53 in heat shock induced cell death in tumor cells, we have identified mutated form of p53 with two functional alleles by reverse transcriptase polymerase chain reaction, and the deletion and addition of nucleotides had resulted in C-terminal deletion of 50 amino acids. We demonstrated that Fas/CD95 induced apoptosis requires p53, and hypothesized that C-terminal deletion and loss of oligomerization domain and nuclear localization signal probably are responsible for $\mathrm{p} 53$-transcritpion independent apoptosis as suggested $[10,11]$. In the present study we show that there are two processed pseudogenes for p53 in this tumor model and one of them also has ribosomal entry site. A comparative genome analysis further revealed that the processed pseudogene is predominantly present in all the rat and mouse species but absent in humans.

\section{MATERIALS AND METHODS}

\subsection{Animal Handling}

All animal maintenance and handling was accomplished as per the institutional ethical committee approval at Centre for Cellular and Molecular Biology, Hyderabad, India. 


\subsection{Tumor Growth and Cell Culture Maintenance}

AK-5 tumor cell line is established from i.p injections of cell-free ascites fluid of a chemically induced and established rat liver tumor, Zajdela ascetic hepatoma (ZAH). These cells possess typical characteristics of macrophages. Single clone of AK-5 tumor, called BC8, was adapted to grow in culture for several generations in Dulbecco's Modified Eagle's Medium (DMEM) with $10 \%$ heat inactivated fetal calf serum (FCS) in the presence of penicillin $(100 \mathrm{U} / \mathrm{ml})$ and streptomycin $(50$ $\mu \mathrm{g} / \mathrm{ml}$ ) is used in the present study. Rat fibroblasts (F111) was procured from ATCC and maintained similar to BC8 as mentioned above. $\mathrm{BC}-8$ cells $\left(8 \times 10^{6}\right.$ cells $)$ were used for injection either for s.c. or i.p. of six-week-old naïve male Wistar rats and tumor growth was monitored. The i.p tumor development approximated by the mean total cell mass calculated from the percentage of packed cells and the total ascites weight.

\subsection{Genomic DNA Isolation}

For normal rat live genomic DNA, six month old male Wistar Rat was scarified as per institutional animal ethics recommendations and genomic DNA was isolated from the liver by phenol: chloroform method and used in the present experiment.

\subsection{RNA Isolation and cDNA Library Construction}

The control and heat stressed tumor cells are subjected to single step total RNA isolation using Trisol reagent, the integrity of RNA was examined by $1 \%$ agarose gel, and $5 \mu \mathrm{g}$ total RNA was used for cDNA preparation by reverse transcriptase system containing the MMLV reverse transcriptase enzyme and oligo $\mathrm{d}(\mathrm{T})$ primer and the cDNA prepared was used for further experiments.

\subsection{Primers Used for the Polymerase Chain Reaction}

PCR primers were designed for the cDNA clone spanning the coding sequence of rat wild type p53 (Acc. No. X13058). Four sets of primers for the amplification of full length as well as partial cDNA amplification were made and used in the present study (Figure 1(a)). Primer set II, P1: Forward-5' atggatccatggaggattcacagtcg 3', P2: Reverse-5' atgaattcgcacagggcatggtcttc 3'; Primer Set III, P3: Forward-5' atggatcctctgccagctggcgaagacat 3', P4: Reverse-5' atgaattcggacaggcacaaacacga 3'; Primer Set IV, P5: Forward-5'atggatcctgaggttcgtgtttgtgc 3', P6: Reverse-5' atgaattctgtcagtctgagtcaggc 3'; and the Primer set $\mathrm{I}$ is the combination of primers $\mathrm{P} 1$ and $\mathrm{P} 6$. Care has been taken while designing the PCR primes to have $50 \%$ $\mathrm{GC}$ content. The PCR conditions are as follows, $94^{\circ} \mathrm{C}$ $1 \mathrm{~min}$ followed by $94^{\circ} \mathrm{C}-1 \mathrm{~min}, 55^{\circ} \mathrm{C}-1 \mathrm{~min}, 72^{\circ} \mathrm{C}-1$ $\min \times 30$ cycles unless otherwise indicated.

\subsection{Southern Blot Analysis}

The full length wild type p53 cDNA $(1.2 \mathrm{~kb})$ was radiolabeled using $\alpha^{32} \mathrm{P}$-dATP by random primer labeling. Hundred nanograms of the template DNA was incubated

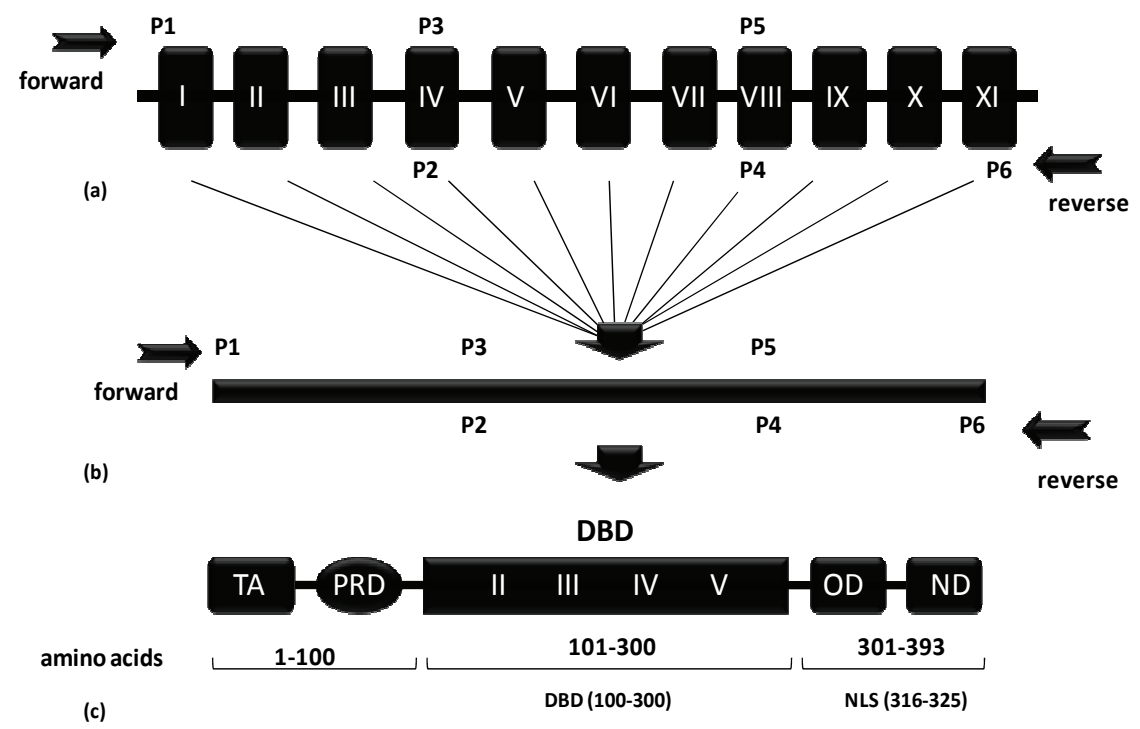

Figure 1. Structural organization of p53. (a) Genome organization depicting the exons of p53 and indicating the primers P1-P6 matching regions; (b) Schematic representation of coding region (cDNA) indicating primers P1-P6; (c) Functional domains showing the conserved regions of p53, appropriate amino acid lengths (1-393) are mentioned. DBD: DNA binding region; NLS: nuclear localization signal. 
$\left(37^{\circ} \mathrm{C}, 15 \mathrm{~min}\right)$ with dNTPs exempting dATP and in the presence of $10 \mu \mathrm{ci}$ of $\alpha^{32} \mathrm{P}-\mathrm{dATP}$, random primer, Klenow enzyme $(5 \mathrm{U})$ and reaction buffer. After the reaction, labeled template was purified through sephadex G-50 column, probe containing $1 \times 10^{8} \mu \mathrm{ci}$ per microgram DNA was used for hybridization. PCR amplicons first run on $1 \%$ agarose gels were vacuum transferred to $\mathrm{N}+$ nylon membrane (Amersham), UV cross-linked and hybridized with radiolabeled probe for overnight. Blots were washed under stringent conditions (sodium phosphate buffer + SDS) and exposed to X-ray film, and photographed.

\subsection{Cloning and Sequence Characterization}

The PCR amplicons are purified using PCR Wizard purification system (Promega, USA) either cloned in TOPO cloning vector and or taken to automated DNA sequence analysis (Model 3730, M/s Applied Biosystems, USA). The obtained DNA sequences were subjected to blast analysis (Entrez at http://www.ncbi. nlm.nih.gov) and the deduced amino acid sequences were analyzed at http:// www.expasy.ch, and http://www. isrec.isb-sib.ch.

\section{RESULTS}

\subsection{Heat Stress Induces p53 Transcription}

In continuation of our interest to know the functional significance of p53 in rat histiocytic tumor models, we compared control cells with heat stress and found that heat stress enhanced p53 transcription (Figure 2(a)). Interestingly when heat stressed samples were subjected for partial PCR analysis we found that primer set II gave two prominent amplicons, while primer sets III and IV giving single amplicon (Figure 2(b)). The PCR amplicons obtained by all the primer sets were excised from agarose gel, purified using PCR product purification kit (PCR Wizard, Qiagen) and re-amplified using same set of primers. All the amplicons showed significant re-amplification suggesting that these amplicons are p53 gene specific (Figure 2(c)). However to confirm and avoid ambiguity with p53 sequence specificity, all the products hybridized with wild type radio labeled p53. Except the lower band of the amplicon with primer set II, all other amplicons showed signficant binding to the radiolabeled probe (Figure $2(\mathbf{d})$ ). The amplicons were cloned in TA cloning vector (Promega) and subjected to automated DNA sequencing. The sequences obtained were aligned with wild type p53 cDNA sequence and found to be homologous (data not shown). While full length did not show any duplication, only primer set II showing such amplicon suggested presence of possible pseudogenes.

\subsection{BC8 Genome Contains a Processed Pseudogene}

In addition to the two alleles reported [10] the additional amplicons obtained may be related to processed p53 alleles originating from the genomic DNA. Therefore the genomic DNA from the tumor cells was isolated and subjected to genomic PCR using p53 cDNA specific primer sets I, II, III, and IV. While primer sets I, II, and IV were giving a single amplicon, primer set III did not yield any amplification (Figure 3(a)). Genomic southern however identified only the full length amplicon amplified using the primer set I (Figure 3(b)). These results therefore suggested a processed pseudogene of p53 in these tumor cells.
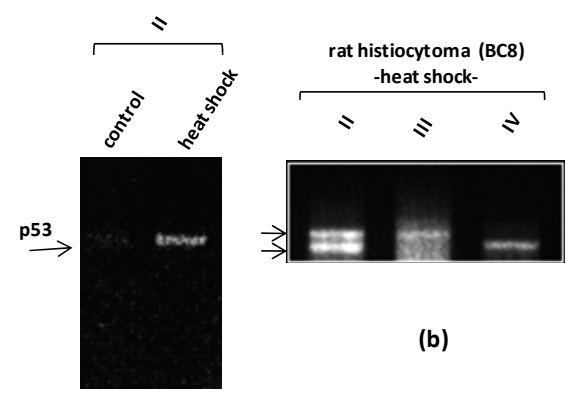

(b)

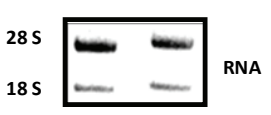

(a)

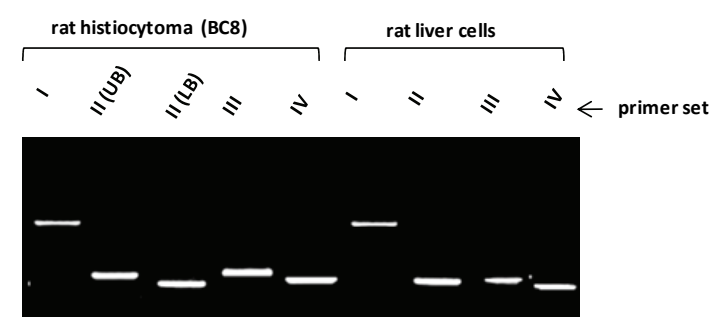

(c)

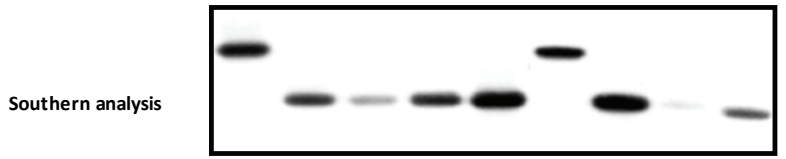

(d)

Figure 2. Reverse transcription and polymerase chain reaction. (a) The total RNA from control and heat shocked BC8 tumor cells was isolated and subjected to RT-PCR analysis with primer set I. The RNA loading control was also shown with intact 28S and 18S RNA; (b) The cDNA of heat shocked BC8 cells was used as a template to amplify p53 with primer sets II, III, and IV. Note only the primer set II showing two amplicons; (c) Re-amplification of first round PCR products after gel elution with appropriate primer sets mentioned; (d) Southern blot analysis of re-amplified PCR products. 


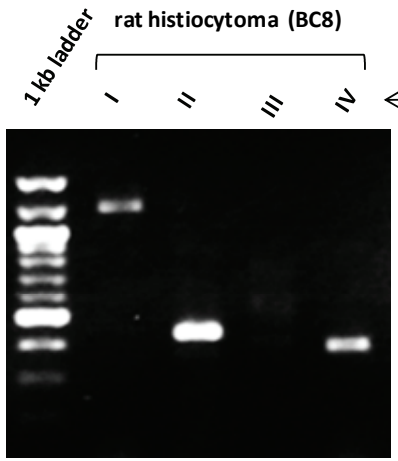

(a)

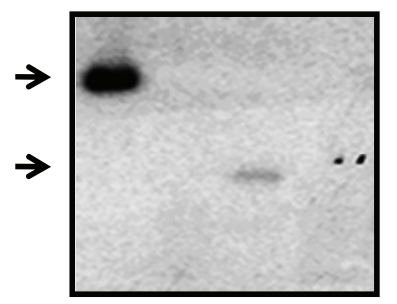

(c)

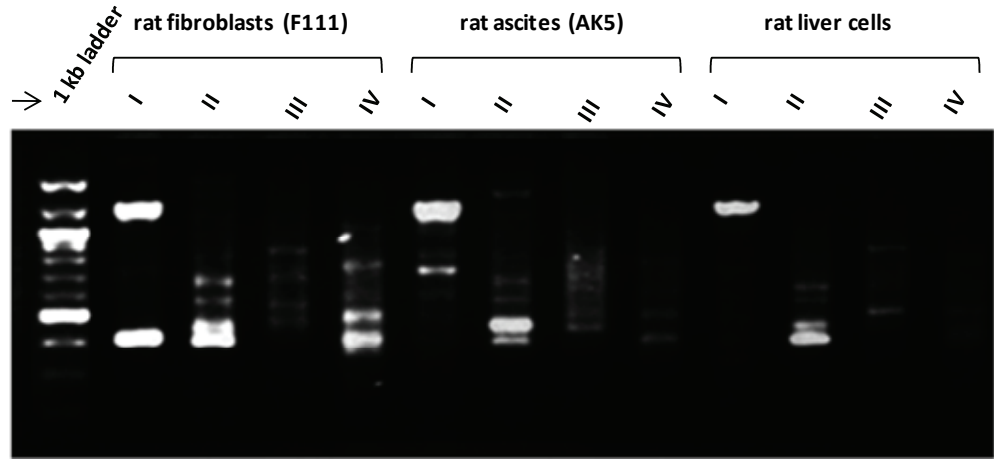

(b)

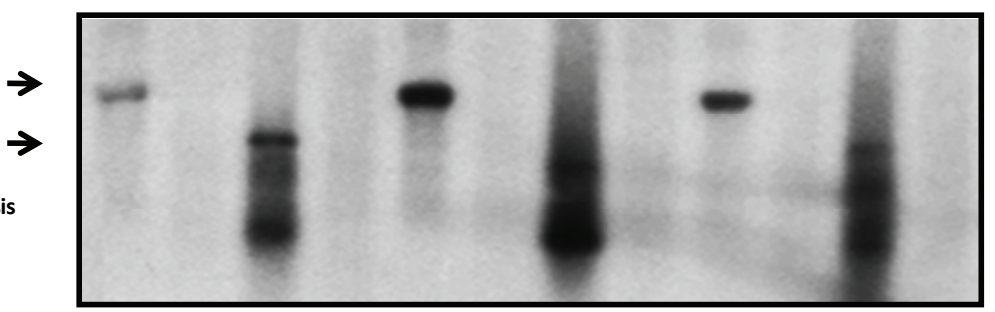

(d)

Figure 3. Genomic PCR and Southern analysis. (a) BC8 genomic PCR analysis with four primer sets, I, II, III, and IV; (b) Genomic Southern analysis of PCR products obtained from Figure 3(a); (c) Genomic PCR analysis of rat fibroblasts, rat histiocytoma (AK5), and rat liver cell; (d) Genomic Southern analysis of PCR products obtained from Figure 3(c).

Processed pseudogenes arise through a mechanism whereby a spliced mRNA is reverse transcribed and subsequently inserted into the genome [12]. If pseudogenes are formed in this way during evolution, these pseudogenes should present in the rat genome and should coexist with all the cell types. To examine this, transformed rat fibroblast cells (F111), parental ascites rat histiocytoma (AK5) were compared with the normal rat genomic DNA.

\subsection{Blast and In-Silico Translational Analysis}

We went ahead of cloning p53 pseudogene and sequence alaysis of cloned product using automated DNA sequencing. From the sequence analysis we found that there is indeed a processed pseudogene having a potential to provide two gene products with different reading frames. A comparative sequence alignment of processed pseudogene with full length RTPCR product of rat histiocytoma additionally showed high sequence homology. Analysis of pseudogene revealed loss of DNA binding region (nt 700-860) and nuclear localization signal (nt 1030-1080) of cDNA (Figure 4). Whole rat genome Blast analysis with cDNA sequence identified its chromosome localization on chromosomes 14,13,10, 9 and 2, and the pseudogene sequence Blast identified its additional localization at chromosome 18 (Table 1).

\section{DISCUSSION}

The p53 gene is frequently lost or rearranged in a large variety of cancers, and most of the alterations in p53 are found in the core domain that interfere with p53 DNAbinding activity [5]. Although p53 has been a wonder molecule and the guardian of genome, mutation of p53 affects its native functions including the antiapoptotic function. Several p53 mutant cells are reported to have lost apoptotic functions but not the cell cycle inhibition $[13,14]$. While our earlier study suggesting that loss of C-terminal 50 amino acids could have played a role in p53-transcription independent apoptosis via Fas/CD95 translocation from golgi to plasma membrane $[11,15]$, a report from Zhu et al. [16] indicated that the N-terminal 43-63 amino acid are more than sufficient to activate p53 transcription dependent apoptosis. Further, induction of pro-apoptotic factor Bax, a known transcriptional client for p53 [17], and subsequent activation of intrinsic apoptotic death pathway through mitochondrial dysfunction [18] directed us to look for possible processed genes in the tumor genome.

By definition, pseudogenes lack a function. However, the classification of pseudogenes generally relies on computational analysis of genomic sequences using complex algorithms [19]. It has been established that quite a few pseudogenes can go through the process of 
transcription, either if their own promoter is still intact or in some cases using the promoter of a nearby gene; this expression of pseudogenes also appears to be tissue-specific [20]. Pseudogenes are often referred to in the scientific literature as nonfunctional DNA. Failure to observe pseudogenes coding for a product under experimental conditions is no proof that they never do so inside an organism. Homologous recombination between the intact functional p53 gene and the p53 pseudogene is thought to have occurred in such a perturbed intracellular environment with genomic instability, thus inactivating the intact allele of the functional p53, therefore the persistence of pseudogenes is in itself additional evidence for their activity. Natural selection would remove

\section{p53 cDNA vs. Pseudogene}

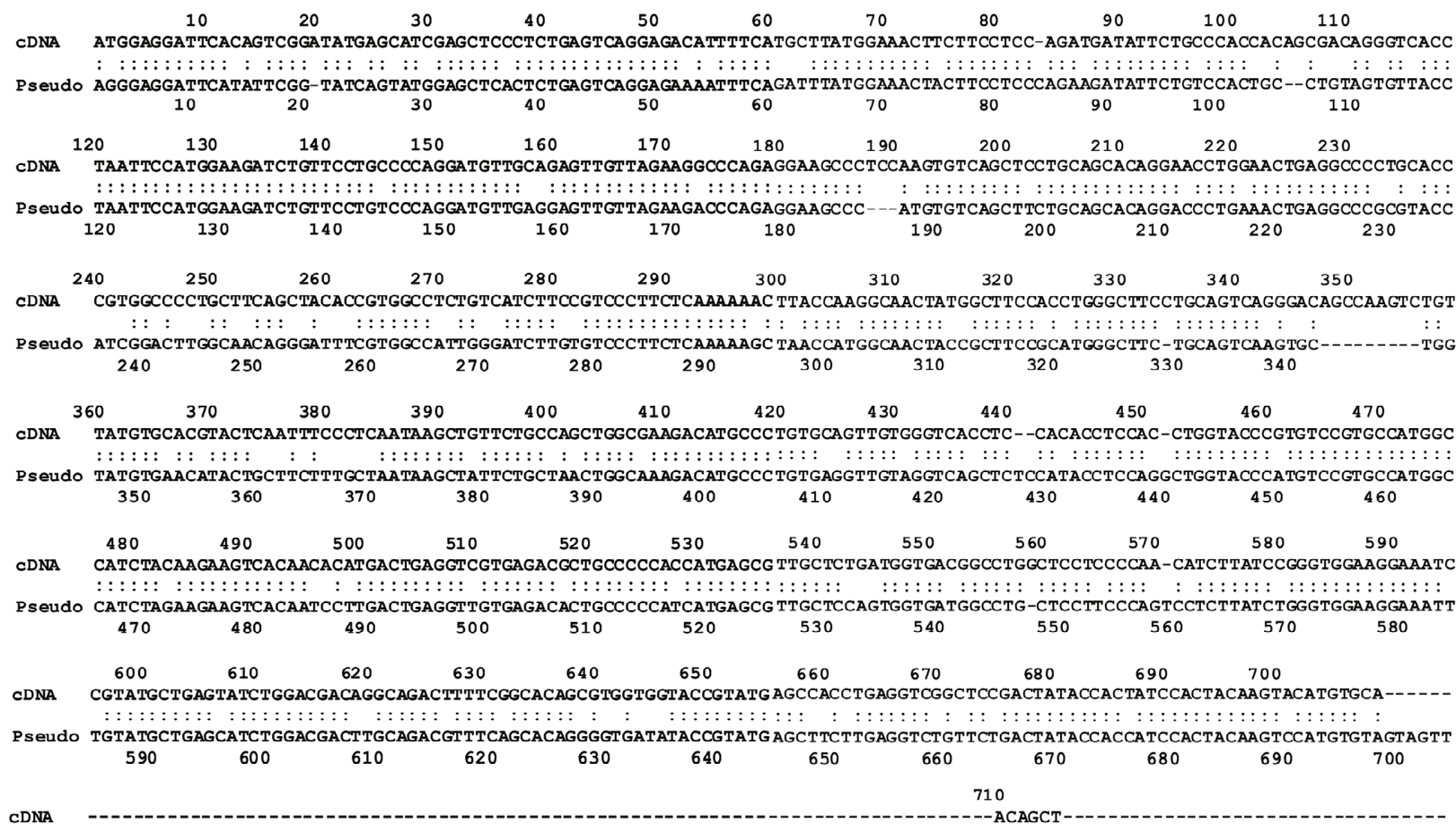

Pseudo CCTACATGGGGACATGACCTGCCTGCCCATCCTTACCATCACCACATTGGAAGATTCCAGTGAAACCTTCCAGGACAGGACAGCTTTGAAGTTCATGTTTTGTGAGGTCTGTTCTGACT

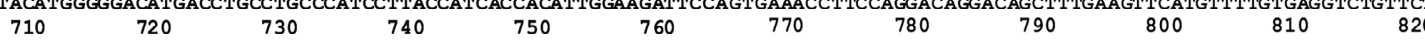
$\begin{array}{llllllll}720 & 730 & 740 & 750 & 760 & 770 & 780 & 790\end{array}$

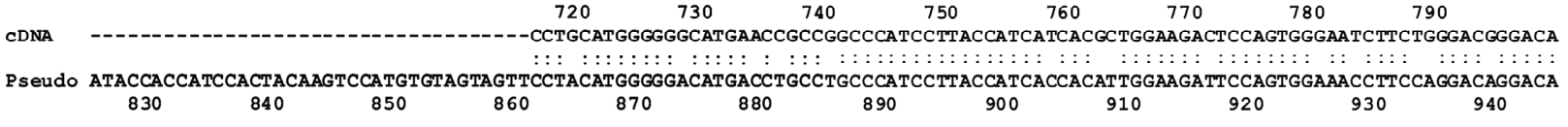
$\begin{array}{llllllllll}830 & 840 & 850 & 860 & 870 & 880 & 890 & 900 & 910 & 920\end{array}$

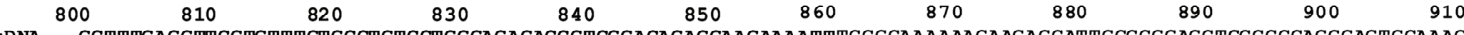

CDNA GCTTTGAGGTTCGTGTTTGTGCCTGTCCTGGGAGAGACCGTCGGACAGAGGAAGAAAATTTCCGCAAAAAAGAAGAGCATTGCCCGGAGCTGCCCCCAGGGAGTGCAAAGAGAGCACTGC $::::::::::::::::::::::: \quad:$

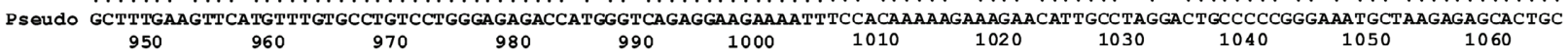

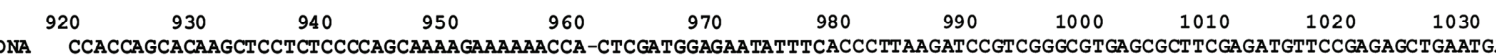

CDNA CCACCAGCACAAGCTCCTCTCCCCAGCAAAAGAAAAAACCA-CTCGATGGAGAATATTTCACCCTTAAGATCCGTCGGGCGTGAGCGCTTCGAGATGTTCCGAGAGCTGAATGAGGCCTT

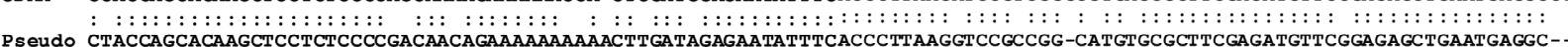

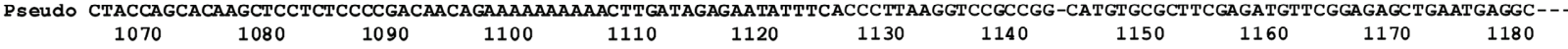
$\begin{array}{llllllllll}1040 & 1050 & 1060 & 1070 & 1080 & 1090 & 1100 & 1110 & 1120 & 1130\end{array}$ CDNA $\begin{array}{cccccccc}1040 & 1050 & 1060 & 1070 & 1080 & 1090 & 1100 & 110\end{array}$

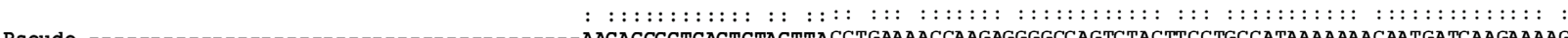

\begin{tabular}{|c|c|c|c|c|c|c|c|c|c|}
\hline & & 1190 & 1200 & 1210 & 1220 & 1230 & 1240 & 1250 & 1260 \\
\hline & 1160 & & & & & & & & \\
\hline CDNA & TGGGGCCTGACTCAGACTGA & & & & & & & & \\
\hline Pseudo & $\begin{array}{l}::::::::::::::::: \\
\text { TGGGGCCTGACTCAGACTGA }\end{array}$ & & & & & & & & \\
\hline
\end{tabular}

Figure 4. Blast analysis of p53 cDNA and pseudogenes showing the loss of DNA binding domain (DBD) and nuclear localization signal (NLS) in the pseudogene. 
Table 1. Genome blast analysis showing the chromosome localization of cDNA and pseudogenes.

\begin{tabular}{|c|c|c|c|c|}
\hline S. No. & Accession number & chromosome & E value & $\%$ identity \\
\hline \multirow[t]{5}{*}{ cDNA } & $\begin{array}{c}\text { NW } 047430.2 \\
\text { NW 001084694.1 }\end{array}$ & 14 & 0.0 & 84 \\
\hline & $\begin{array}{c}\text { NW } 047390.2 \\
\text { NW } 001084680.1\end{array}$ & 13 & 0.0 & 78 \\
\hline & $\begin{array}{c}\text { NW } 047334.2 \\
\text { NW 0010884656.1 }\end{array}$ & 10 & 1e-140 & 100 \\
\hline & $\begin{array}{c}\text { NW 047813.2 } \\
\text { NW 001084694.1 }\end{array}$ & 9 & 0.0 & 84 \\
\hline & $\begin{array}{c}\text { NW } 047627.2 \\
\text { NW } 001084680.1\end{array}$ & 2 & 0.0 & 87 \\
\hline \multirow[t]{5}{*}{ pseudogene } & $\begin{array}{c}\text { NW } 047518.2 \\
\text { NW 001084740.1 }\end{array}$ & 18 & 0.0 & 99 \\
\hline & $\begin{array}{c}\text { NW 047430.2 } \\
\text { NW 001083694.1 }\end{array}$ & 14 & 0.0 & 84 \\
\hline & $\begin{array}{c}\text { NW } 047390.2 \\
\text { NW 001084680.1 }\end{array}$ & 13 & $3 e-23$ & 76 \\
\hline & $\begin{array}{c}\text { NW 047334.2 } \\
\text { NW 001084656.1 }\end{array}$ & 10 & $1 e-42$ & 81 \\
\hline & $\begin{array}{c}\text { NW 47813.2 } \\
\text { NW 001084880.1 }\end{array}$ & 9 & $5 e-86$ & 95 \\
\hline
\end{tabular}

this type of DNA if it were useless, since DNA manufactured by the cell is energetically costly. As the function of more pseudogenes is being uncovered by testable and repeatable science, it is evident that these genetic elements, which are copiously spread in the genomes of different organisms, have been created with purpose.

In addition, and in contrast to previously believed information that pseudogenes are non functional copies of genes [21,22], growing evidence suggests that at least some pseudogenes are functional. It has been demonstrated that pseudogenes notably arise from seemingly absent or disabled promoters, premature stop codons, splicing errors, frameshift-causing deletions and insertions, etc., and do not necessarily abolish gene expression [23,24]. McCarrey et al. [25] have suggested that pseudogenes can be functional in terms of the regulation of the expression of its paralogous genes, otherwise antisense to pseudogenes should not interfere with cellular functions. In support of this earlier we have used $\mathrm{N}$ terminal siRNA to p53 and could inhibit its functions [10]. With respect to the evolution of regulatory functions of pseudogenes we must now conclude that transcribed pseudogenes are not necessarily without function. Indeed, they would appear to be especially suited to roles involving the antisense regulation of the active genes to which they are related [24]. In summary we report a processed pseudogene and additional translational products for $\mathrm{p} 53$ in a rat histiocytoma that differ from the parental tumor and from the rat genome may have function roles upon stress and tumorigenesis.

\section{REFERENCES}

[1] Green, D.R. and Kroemer, G. (2009) Cytoplasmic functions of the tumour suppressor p53. Nature, 458(7242), $1127-1130$.

[2] Darzynkiewicz, Z. (1995) Apoptosis in antitumor strategies: modulation of cell cycle or differentiation. Journal of Cellular Biochemistry, 58(2), 151-159.

[3] Halevy, O., Michalovitz, D. and Oren, M. (1990) Different tumor-derived p53 mutants exhibit distinct biological activities. Science, 250(4977), 113-116.

[4] Michalovitz, D., Halevy, O. and Oren, M. (1991) P53 mutations-gains or losses. Journal of Cellular Biochemistry, 45(1), 22-29.

[5] Levine, A.J. (1997) P53, the cellular gatekeeper of growth and division. Cell, 88(3), 323-331.

[6] Weghorst, C.M., Buzard, G.S., Calvert, R.J., Hulla, J.E. and Rice, J.M. (1995) Cloning and sequence of a processed p53 pseudogene from rat: A potential source of false 'mutations' in PCR fragments of tumor DNA. Gene, 166(2), 317-322.

[7] de Fromentel, C.C. and Soussi, T. (1992) TP53 Tumor suppressor gene: A model for investigating human mutagenesis. Genes Chromosomes and Cancer, 4(1), 115.

[8] El-Deiry, W.S., Kern, S.E., Pietenpol, J.A., Kinzler, K.W. and Vogelstein, B. (2002) Definition of a consensus binding site for p53. Nature Genetics, 1(1), 45-49. 
[9] Blagosklonny, M.V. and El-Deiry, W.S. (1998) Acute overexpression of wt p53 facilitates anticancer druginduced death of cancer and normal cells. International Journal of Cancer, 75(6), 933-940.

[10] Sreedhar, A.S., Pardhasaradhi, B.V.V., Khar A. and Srinivas, U.K. (2002) Effect of C-terminal deletion of P53 on heat induced CD95 expression and apoptosis in a rat histiocytoma. Oncogene, 21(25), 4042-4049.

[11] Bennett, M., Macdonald, K., Chan, S.W., Luzio, J.P., Simari, R. and Weissberg, P. (1998) Cell surface trafficking of Fas: A rapid mechanism of p53-mediated apoptosis. Science, 282(5387), 290-293.

[12] Lewin, B. (1990) Structural genes evolve in families. In: Genes IV, Oxford University Press, New York, 497-517.

[13] Rowan, S., Ludwig, R.L., Haupt, Y., Bates, S., Lu, X., Oren, M. and Vousden, K.H. (1996) Specific loss of apoptotic but not cell-cycle arrest function in a human tumor derived p53 mutant. EMBO Journal, 15(4), 827838.

[14] Ryan, K.M. and Vousden, K.H. (1998) Characterization of structural p53 mutants which show selective defects in apoptosis but not cell cycle arrest. Molecular Cell Biology, 18(7), 3692-3698.

[15] Sreedhar, A.S., Pardhasaradhi, B.V.V., Khar, A. and Srinivas, U.K. (2000) Heat induced expression of CD95 and its correlation with the activation of apoptosis upon heat shock in rat histiocytic tumor cells. FEBS Letters, 472(2-3), 271-275.

[16] Zhu, J., Zhou, W., Jiang, J. and Chen, X. (1998) Identification of a novel p53 functional domain that is necessary for mediating apoptosis. Journal of Cellular Biochemistry, 273(21), 13030-13036.
[17] Miyashita, T. and Reed, J.C. (1995) Tumor suppressor p53 is a direct transcriptional activator of the human bax gene. Cell, 80(2), 293-299.

[18] Sreedhar, A.S., Pardhasaradhi, B.V.V., Khar, A. and Srinivas, U.K. (2002) A cross talk between cellular signalling and cellular redox state during heat-induced apoptosis in a rat histiocytoma. Free Radical Biology and Medicine, 32(3), 221-227.

[19] Harrison, P.M., Zheng, D., Zhang, Z., Carriero, N. and Gerstein, M. (2005) Transcribed processed pseudogenes in the human genome: An intermediate form of expressed retrosequence lacking protein-coding ability. Nucleic Acids Research, 33(8), 2374-2383.

[20] Zheng, D., Frankish, A., Baertsch, R., Kapranov, P., Reymond, A., Choo, S.W., Lu, Y., Denoeud, F., Antonarakis, S.E., Snyder, M., Ruan, Y., Wei, C.L., Gingeras, T.R., Guigó, R., Harrow, J. and Gerstein, M.B. (2007) Pseudogenes in the ENCODE regions: Consensus annotation, analysis of transcription, and evolution. Genome Research, 17(6), 839-8351.

[21] Zhang, Z.D., Frankish, A., Hunt, T., Harrow, J. and Gerstein, M. (2010) Identification and analysis of unitary pseudogenes: Historic and contemporary gene losses in humans and other primates. Genome Biology, 11(3), R26.

[22] Torrents, D., Suyama, M., Zdobnov, E. and Bork, P. (2003) A genome-wide survey of human pseudogenes. Genome Research, 13(12), 2559-2567.

[23] Woodmorappe, J. (2003) The potential immunological functions of pseudogenes and other 'junk' DNA. Journal of Creation, 17(3), 102-108.

[24] Woodmorappe, J. (2003) Pseudogene function: Regulation of gene expression. Journal of Creation, 17(1), 47-52. 